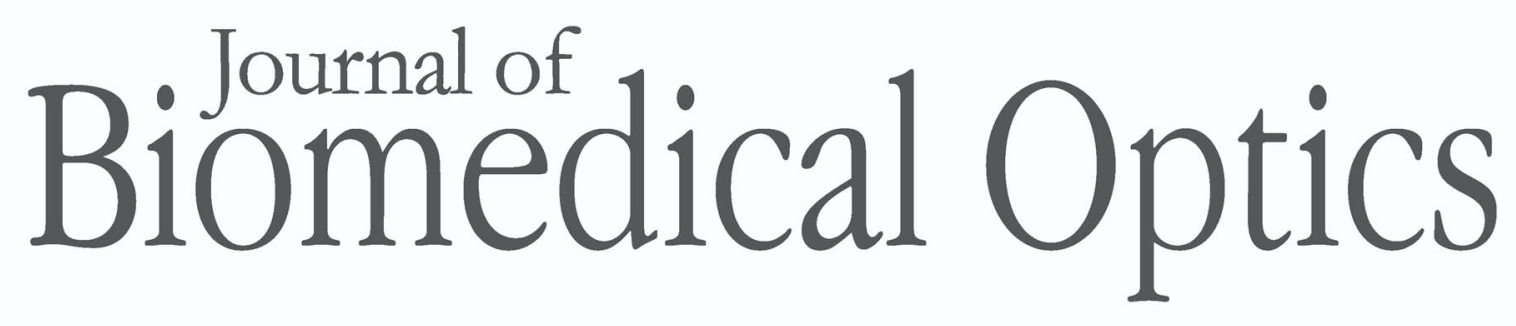

\title{
Morphologic tomography of nonspherical particles using multispectral diffusing light measurements
}

Mohammad Reza Hajihashemi

Xiaoqi Li

Huabei Jiang 


\title{
Morphologic tomography of nonspherical particles using multispectral diffusing light measurements
}

\author{
Mohammad Reza Hajihashemi, Xiaoqi Li, and Huabei Jiang \\ University of Florida, Department of Biomedical Engineering, Gainesville, Florida 32611-6131
}

\begin{abstract}
A series of phantom experiments are conducted to demonstrate the ability of a T-matrix-based inverse algorithm for tomographic recovery of morphologic characteristics of nonspherical particles embedded in heterogeneous turbid media. Diffusely scattered light at several wavelengths along the boundary of the phantom are collected and analyzed to allow for simultaneous extraction of the size, concentration, and aspect ratio of the spheroidal particles. ( 12011 Society of Photo-Optical Instrumentation Engineers (SPIE). [DOI: 10.1117/1.3650313]
\end{abstract}

Keywords: particle characterization; nonspherical particles; tomography.

Paper 11404R received Jul. 26, 2011; revised manuscript received Sep. 12, 2011; accepted for publication Sep. 21, 2011; published online Nov. 2, 2011.

\section{Introduction}

Morphologic characterization of particles inside tissues and organs is the subject of great interest for biomedical applications. Optical imaging techniques are promising candidates for such purpose since they are noninvasive and relatively inexpensive. ${ }^{1-5}$ The basic idea is to analyze the spectrum of scattered light in order to extract morphologic information regarding the particles involved. In Ref. 1, a Mie theory-based inverse technique was employed to retrieve the mean size and the concentration of breast cancer cells using multispectral diffuse optical tomography measurements. In Ref. 2, a light-scattering spectroscopybased technique was proposed for characterizing the sizes of leukemic cells. In Ref. 3, optical measurements were performed to retrieve information regarding the size and shape of peripheral blood particles. In Ref. 4, a Mie theory-based technique was proposed to compute light scattering from biological cells. In Ref. 5, the T-matrix method was utilized to recover the size and aspect ratio of spheroidal cells using angle-resolved backscattering data.

Most of the reported works focused on the study of spherical particles using the Mie scattering model for this purpose. ${ }^{6}$ The drawback of the Mie scattering model is its limited applicability to real particle characterization applications.

Attempts have been made to characterize spheroidal particles in tissue/scattering media using low-coherence interferometry or light-scattering spectroscopy. ${ }^{7-9}$ The aspect ratios of aerosol particles were retrieved using simultaneous measurements of extinction coefficient and phase function. ${ }^{10}$ In Ref. 11 , retrieving the aspect ratio of spheroidal particles was discussed using linear depolarization of the scattered light in the forward and backscatter directions. Phantom experiments were performed in Ref. 12 to retrieve the size and the aspect ratio of spheroidal particles using angle-resolved low-coherence interferometry data. While promising, these methods do not show their ability to retrieve the concentration of the particles, nor are they applicable to an aggregate of randomly oriented particles with different

Address all correspondence to: Mohammad Reza Hajihashemi, University of Florida, J218 BMS Building, Gainesville, Florida 32611-6131; Tel: 352-273-9333. E-mail: mhajihashemi@bme.ufl.edu. sizes. In particular, these methods are inadequate to analyze spheroidal particles located deep in tissue. In this work, we present an inverse algorithm, based on multispectral diffuse optical tomography (MSDOT) coupled with the T-matrix method, to extract the statistical morphologic parameters of randomly oriented spheroidal particles suspended deeply in heterogeneous turbid media.

In MSDOT, near-infrared light, at several wavelengths, is transmitted into the phantom and then the recorded photon flux at its boundary is analyzed to retrieve the images of the absorption and reduced scattering coefficients using the photon diffusion equation-based nonlinear reconstruction algorithm. ${ }^{13,14}$ MSDOT is an emerging modality that provides spectroscopic imaging of deep tissue absorption and scattering coefficients, ${ }^{15-18}$ while the T-matrix method is a generalized Mie-Lorenz theory for calculation of scattering from single or an aggregate of nonspherical particles. ${ }^{19}$ We use a series of phantom experiments to show the ability of our inverse algorithm to simultaneously recover three statistical parameters of elongated polymer microspheres embedded in scattering medium including the concentration, mean size, and aspect ratio. To our knowledge, this work is the first reporting the application of MSDOT for retrieving the statistical parameters of nonspherical particles in turbid media using experimental data.

\section{Inversion Algorithm}

We have derived the following expression which relates the multispectral reduced scattering coefficient of the media, $\mu_{s}^{\prime}(\lambda)$, measured by MSDOT, to the mean size, concentration, and aspect ratio of the contained particles under Gaussian particle size distribution: ${ }^{20}$

$$
\begin{aligned}
\mu_{s}^{\prime}(\lambda)= & \phi \int_{r=r_{\min }}^{r=r_{\max }} \int_{\varepsilon=\varepsilon_{\min }}^{\varepsilon=\varepsilon_{\max }} \frac{e^{-1 / 2\left[\left(r-r_{m}\right)^{2} / \delta_{m}^{2}+\left(\varepsilon-\varepsilon_{m}\right)^{2} / \delta_{\varepsilon}^{2}\right]}}{2 \pi \delta_{r} \delta_{\varepsilon}} \\
& \times\left[1-\frac{\alpha_{1}^{1}(r, \varepsilon)}{3}\right]\left\langle C_{s c a}(r, \varepsilon)\right\rangle d r d \varepsilon
\end{aligned}
$$

1083-3668/2011/16(11)/116014/7/\$25.00 @ 2011 SPIE 

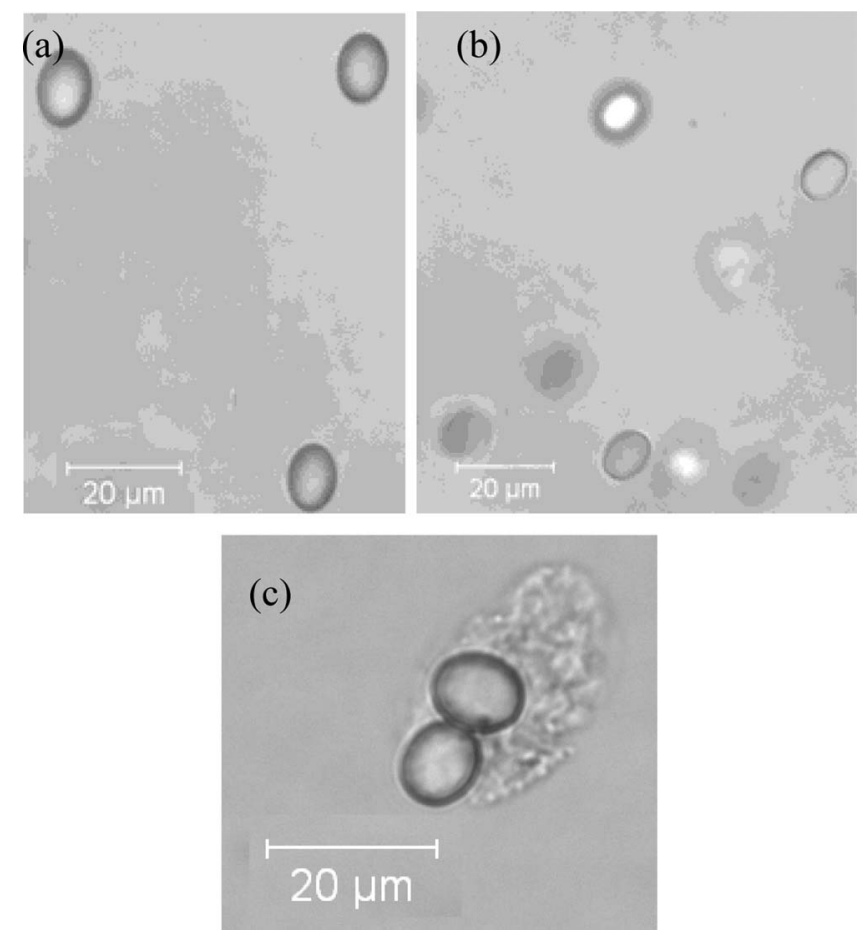

Fig. 1 Microscopy of (a) polystyrene particles inside PDMS. (b) PMMA particles inside PDMS. (c) Suspended PMMA particles recovered from PDMS matrix.

where $r$ and $\varepsilon$ are, respectively, the particle equivalent radius and aspect ratio with $r_{m}$ and $\varepsilon_{m}$ representing their mean value. $r$ is defined as the radius of a sphere having equivalent surface area or volume as a spheroid, while $\varepsilon$ is the ratio of the equatorial radius to the polar radius, which is less than 1 for the prolate spheroidal particles used in this work. The concentration $\phi$ represents the number of particles per $\mathrm{mm}^{3} . \delta_{r}$ and $\delta_{\varepsilon}$ are the standard deviations of the particle size and aspect ratio, respectively. $\left\langle C_{s c a}(r\right.$, $\varepsilon)\rangle$ denotes the averaged scattering cross section of an ensemble of randomly-oriented monodisperse spheroidal particles, having an equivalent radius of $r$ and aspect ratio of $\varepsilon . \alpha_{1}^{1}(r, \varepsilon)$ is a coefficient obtained by expansion of the phase function. ${ }^{19}$ An iterative Newton method, accompanied with Tikhonov-Marquardt regularization, is used to update $r_{m}, \varepsilon_{m}$, and $\phi$ until the minimization between the measured (by MSDOT) and calculated [by Eq. (1)] scattering spectra is reached. If the vector $\Delta \chi$ is the difference between the observed and calculated reduced scattering coefficient, the updates of unknown parameters $\Delta \zeta$ (i.e., $r_{m}$, $\varepsilon_{m}$, and $\phi$ ) are calculated by solving the following matrix equation:

$$
\left(J^{T} J+\gamma I\right) \Delta \zeta=J^{T} \Delta \chi
$$

where $I$ is the identity matrix and $\gamma$ is an appropriately chosen regularization parameter. The elements of the Jacobian matrix $J$ are determined by direct differentiation of Eq. (1) with respect to the model parameters.

\section{Configuration of Phantom Experiments}

To validate the inverse algorithm, we used two types of polymer particles, polystyrene and polymethyl-methacrylate (PMMA), with indices of refraction $\sim 1.57$ to 1.59 and $\sim 1.48$, respectively,

\section{DOT Measurement Sensors}

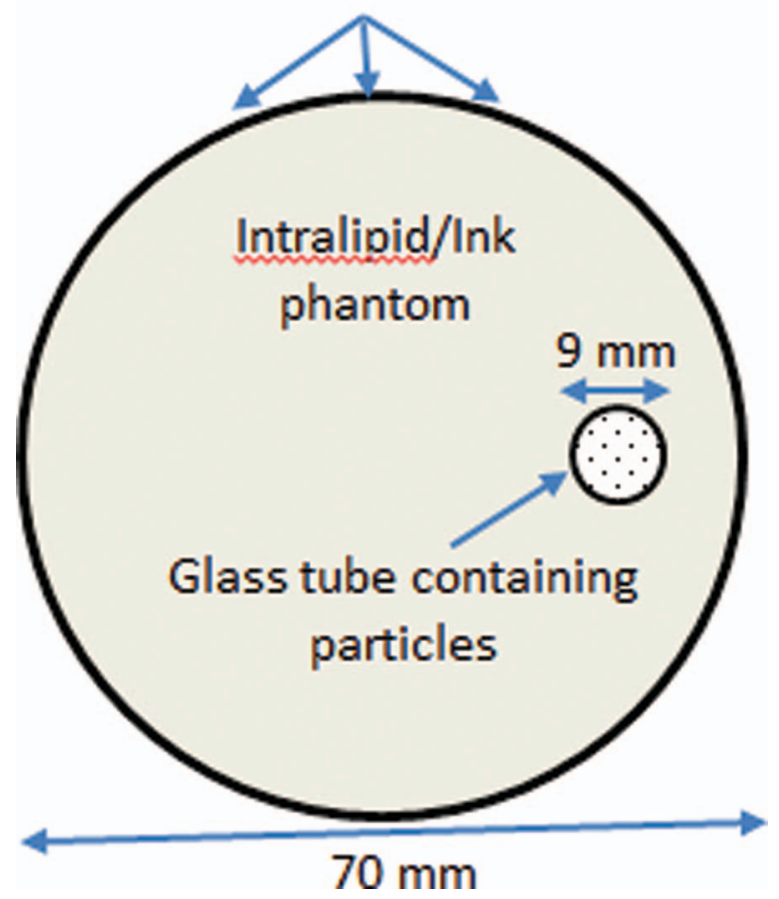

Fig. 2 Diagram of MSDOT measurement system and the phantom experiment.

which are similar to those of tissue. ${ }^{21}$ Both the original polymer microspheres needed to be deformed to become nonspherical. Following the method described in Ref. 22, the particles were embedded in an elastic polydimethylsiloxane (PDMS) matrix. After PDMS was cured, it was sliced into thin strips. The PDMS strips were placed in an oven and heated above the glass transition temperature of the microspheres $\left(\sim 190^{\circ} \mathrm{C}\right)$. While the sample was heated, the microspheres were elongated under uniaxial tension applied to the ends of strips. The sample was then cooled down while the tension was still being applied. Finally, elongated particles were extracted by chemical degradation of the silicon matrix using sodium methoxide in isopropanol-hexane. ${ }^{22}$ Figures 1(a) and 1(b) show the microscopic images of the elongated polystyrene and PMMA particles inside PDMS, while Fig. 1(c) presents the microscopy of the recovered particles after chemical degradation.

As shown in Figs. 1(a) and 1(b), polystyrene particles demonstrate higher elongation (smaller aspect ratio) compared with PMMA particles. The minimum aspect ratio obtained for polystyrene particles was $\sim 0.7$ while it was $\sim 0.8$ for PMMA particles. We then conducted phantom experiments using our MSDOT system at six wavelengths: 690, 733, 775, 808, 840, and $915 \mathrm{~nm} \cdot{ }^{13,14}$ The T-matrix database was generated for particles varying in the range of 0.5 to $6 \mu \mathrm{m}$ in radius, and 0.5 to 1.5 in aspect ratio. ${ }^{19,20}$ The background was a $35-\mathrm{mm}$ radius cylindrical solid phantom consisting of Intralipid and India ink, and had an absorption coefficient of $0.005 \mathrm{~mm}^{-1}$ and a reduced scattering coefficient of $\sim 0.3 \mathrm{~mm}^{-1}$.

A thin glass 9-mm diameter tube containing the nonspherical particles was embedded off-center in the background phantom as shown in Fig. 2. Both the original polystyrene and PMMA microspheres had a nominal diameter of $10 \mu \mathrm{m}$. 
(a) Mean Particle Size

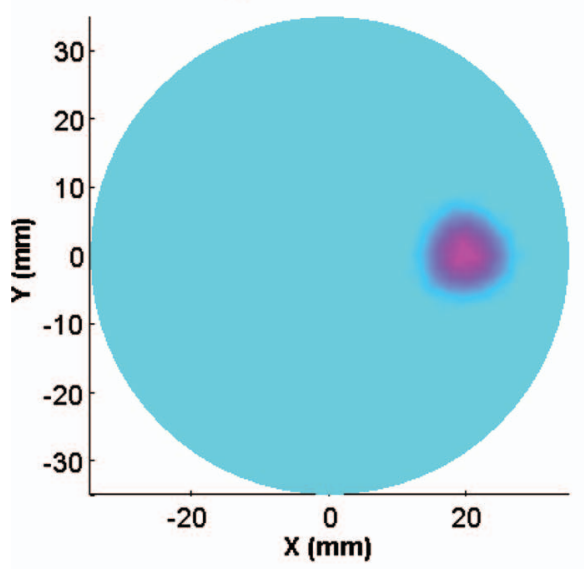

(c) Volume fraction $(\%)$

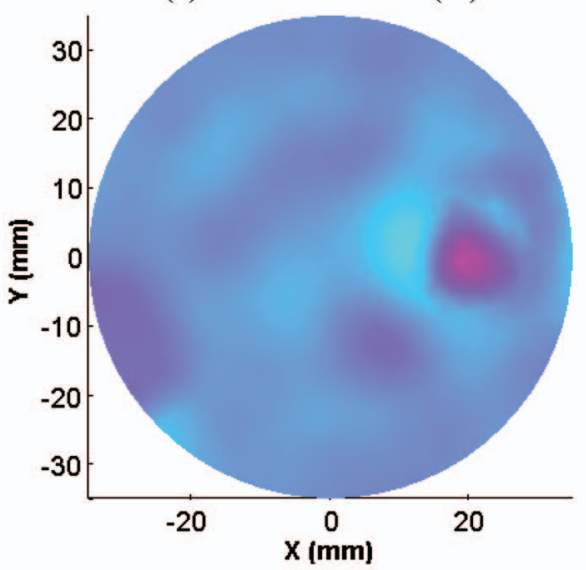

(e) Mean Aspect ratio

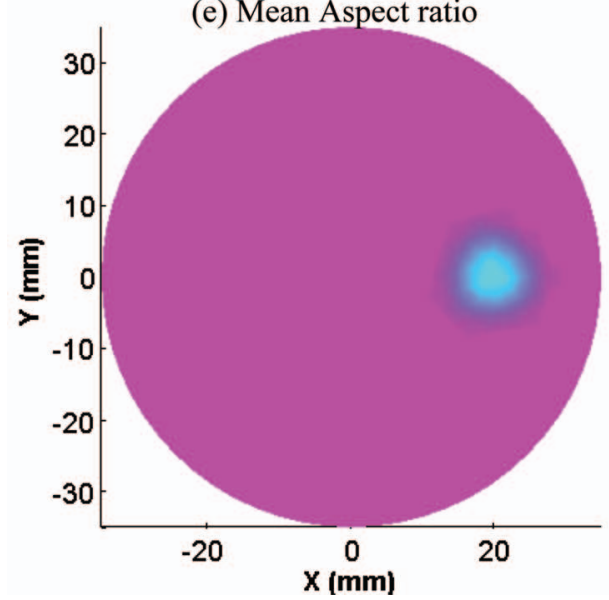

(b) Mean Particle Size $(\mu \mathrm{m})$ along $\mathrm{y}=0$ line
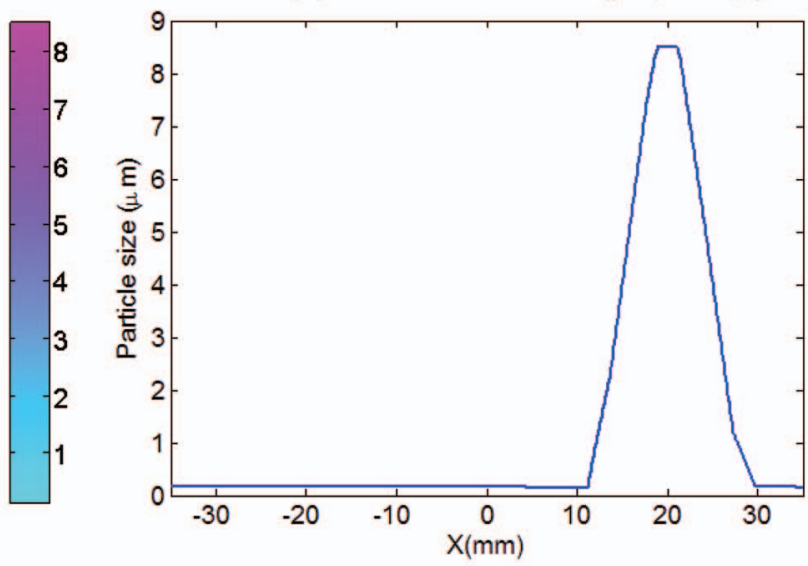

(d) Volume fraction (\%) along $y=0$ line.
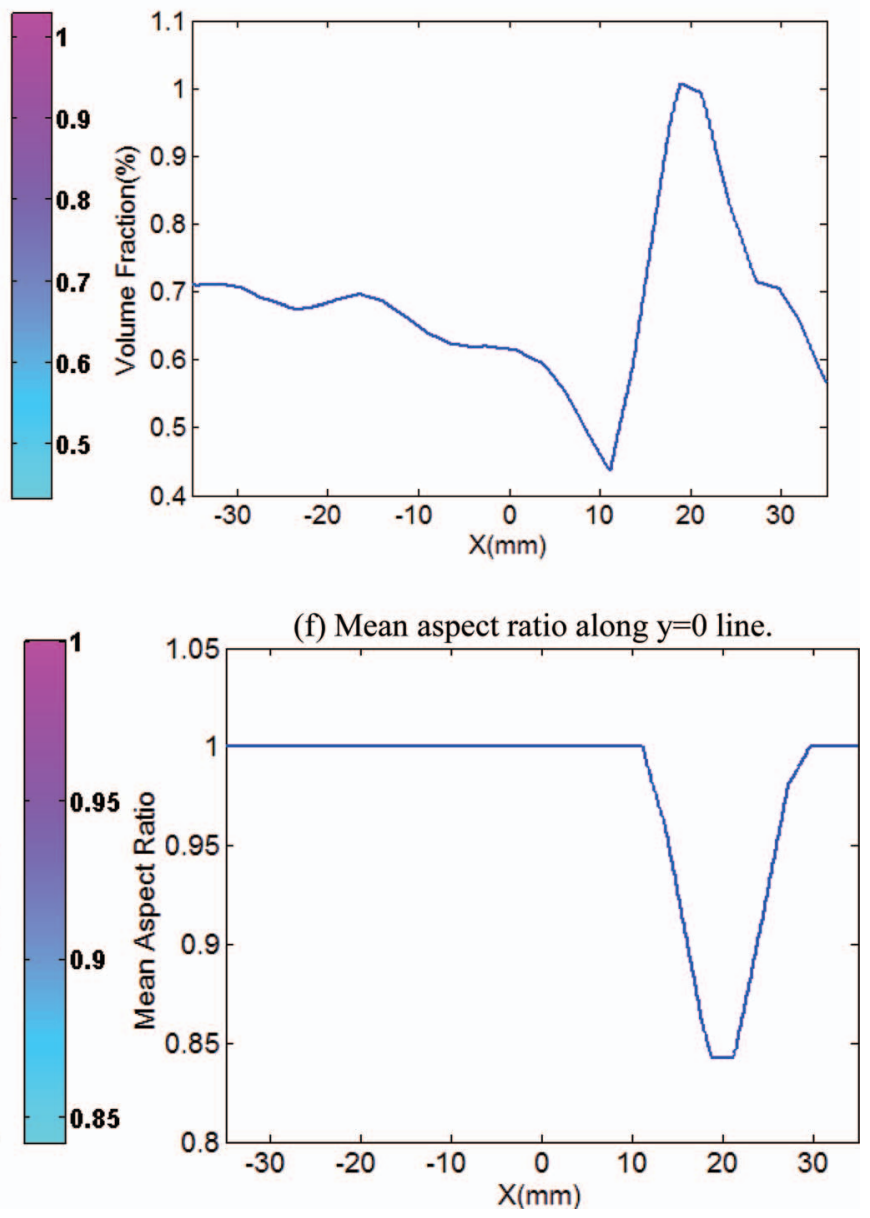

Fig. 3 (a) and (b) Reconstructed particle size, (c) and (d) concentration, and (e) and (f) aspect ratio of PMMA prolate particles.

The details of data collection system are elaborated in Ref. 14. Briefly, light from one of the five continuous-wave laser modules was transmitted to 16 preselected points at the surface of the phantom through an optical switch for 2D (two-dimensional) imaging experiments. $16 \times 16$ measured data was then input into our MSDOT reconstruction algorithm to generate a 2D crosssectional image of the phantom.

It is well known that the main contribution of scattering in the Intralipid background region arises from soybean oil par- ticles, while in the target region the scattering is caused by polymer particles. ${ }^{6,23}$ The Intralipid particles are spherical and have much smaller sizes (less than $500 \mathrm{~nm}$ in diameter) compared with PMMA and polystyrene particles. ${ }^{23}$ Therefore, it is necessary to use separate initial particle values for particle characterization in the background region. ${ }^{6}$ We have generated a second T-matrix database in the range of 20 to $500 \mathrm{~nm}$ for the characterization of the spherical particles in the background region. 
(a) Mean Particle Size

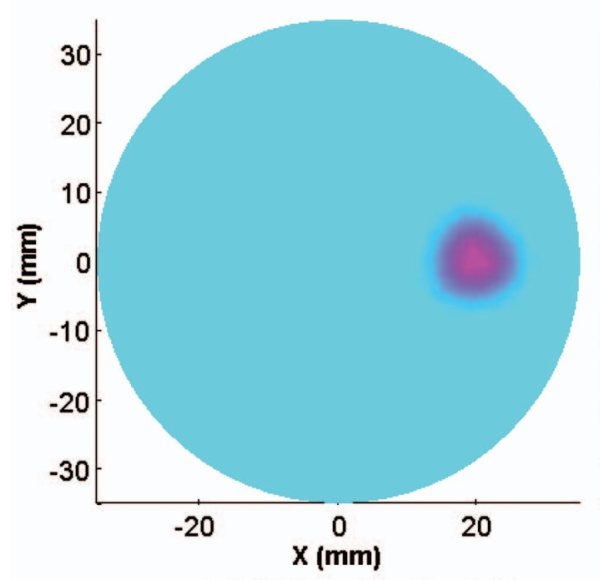

(c) Volume fraction (\%)

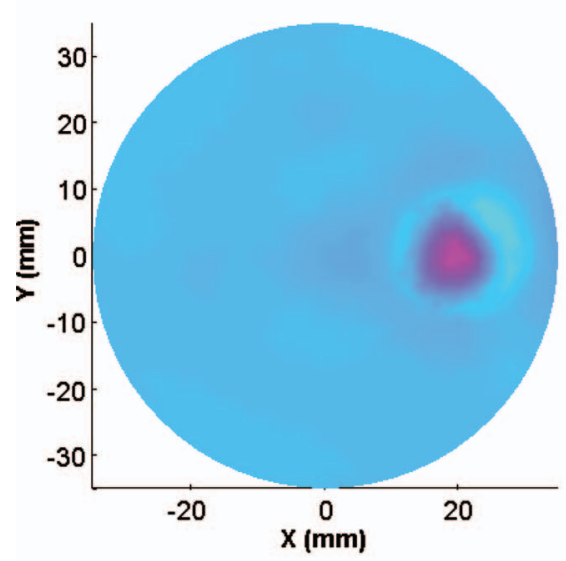

(e) Mean Aspect ratio

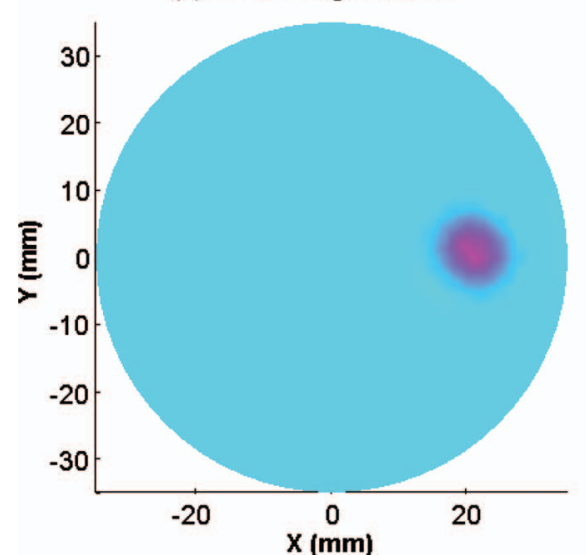

(b) Mean Particle Size $(\mu \mathrm{m})$ along $\mathrm{y}=0$ line
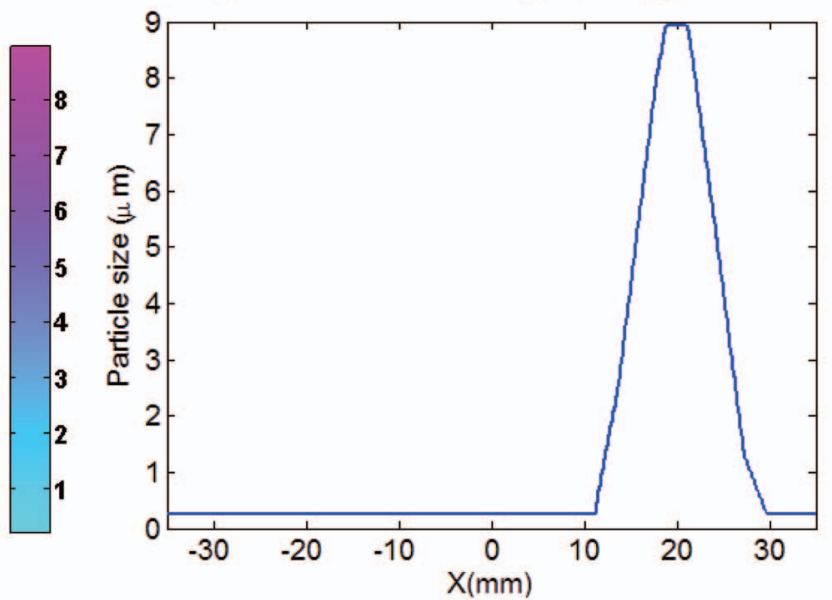

(d) Volume fraction (\%) along $\mathrm{y}=0$ line.
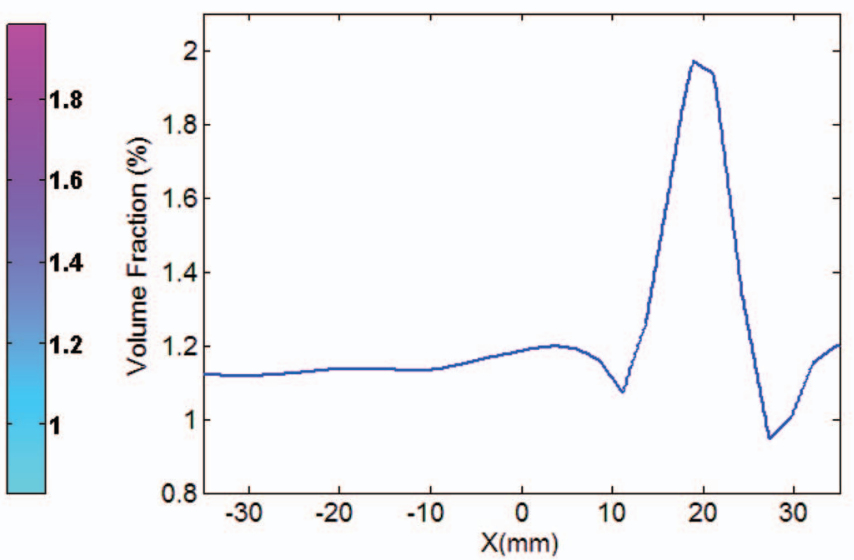

(f) Mean aspect ratio along $\mathrm{y}=0$ line.
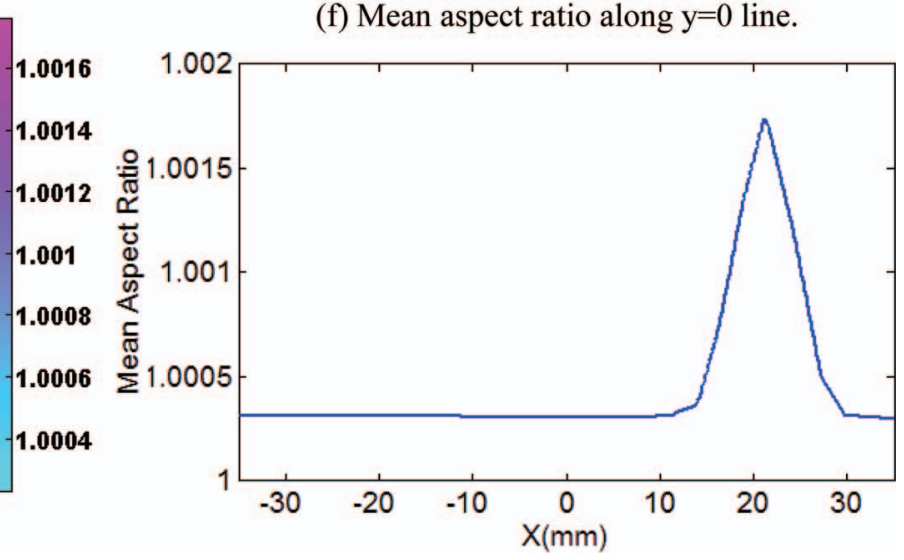

Fig. 4 (a) and (b) Reconstructed particle size, (c) and (d) concentration, and (e) and (f) aspect ratio of PMMA spherical particles.

For in vivo experiments, generally some a priori information is available regarding the types of tissues and the particles involved. If the parameters of scattering particles are considerably different from each other, we will attempt to use different initial parameter values because the scattering images provided by MSDOT will allow us to differentiate different tissue types before we characterize the particles in tissue. However, if the scattering particles are on the same order of size, e.g., in the breast tissue, single initialization in the entire computational domain can reasonably retrieve the unknown parameters.

In all of the presented results, a search algorithm is first used to find appropriate initial values of the unknown parameters using all possible combinations of size, concentration, and aspect ratio. Then, the inverse algorithm is employed to recover the particle morphology by minimizing the error between the measured and computed scattering spectra. ${ }^{20}$ The indices of refraction of 
(a) Mean Particle Size

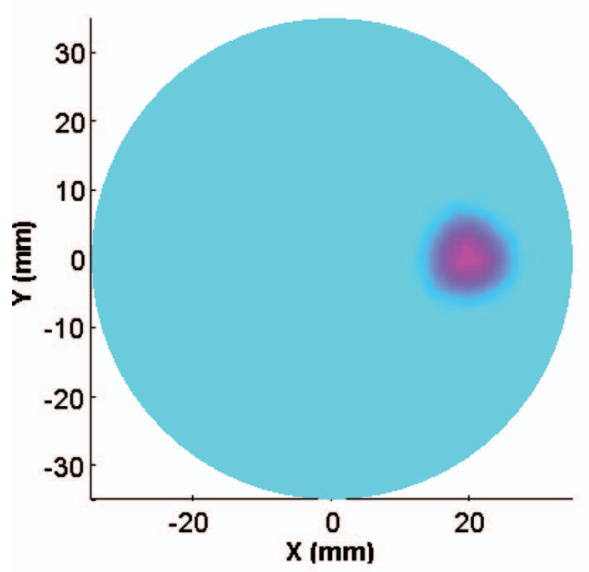

(c) Volume fraction (\%)

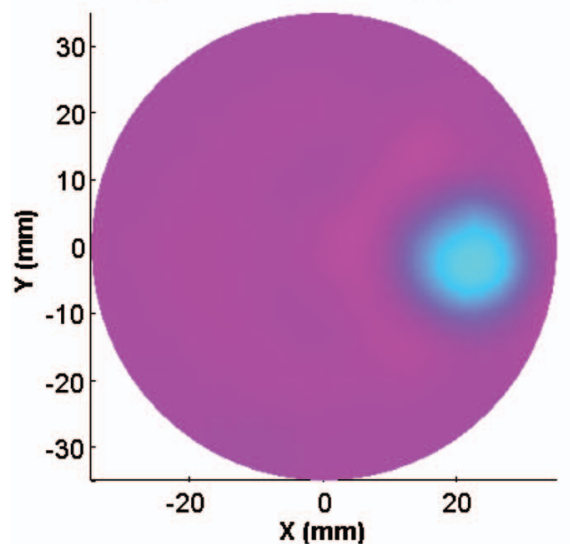

(e) Mean Aspect ratio

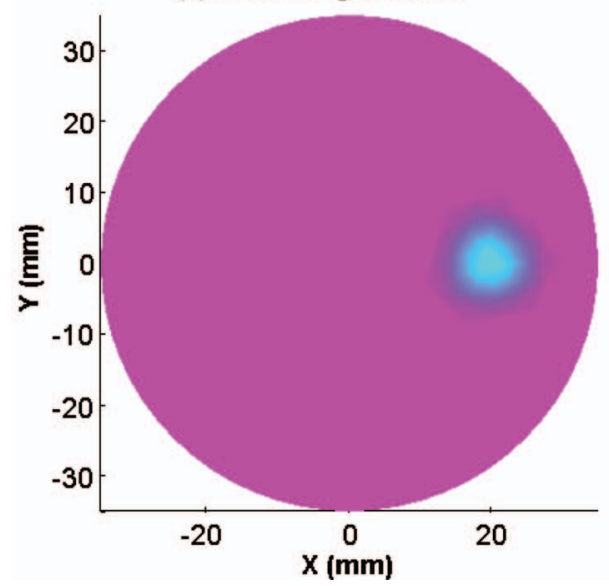

(b) Mean Particle Size $(\mu \mathrm{m})$ along $\mathrm{y}=0$ line

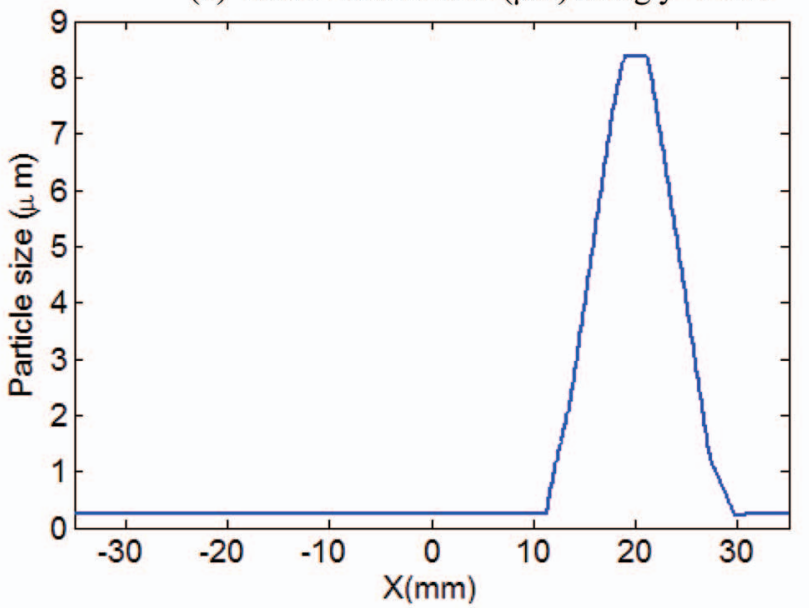

(d) Volume fraction (\%) along $\mathrm{y}=0$ line.
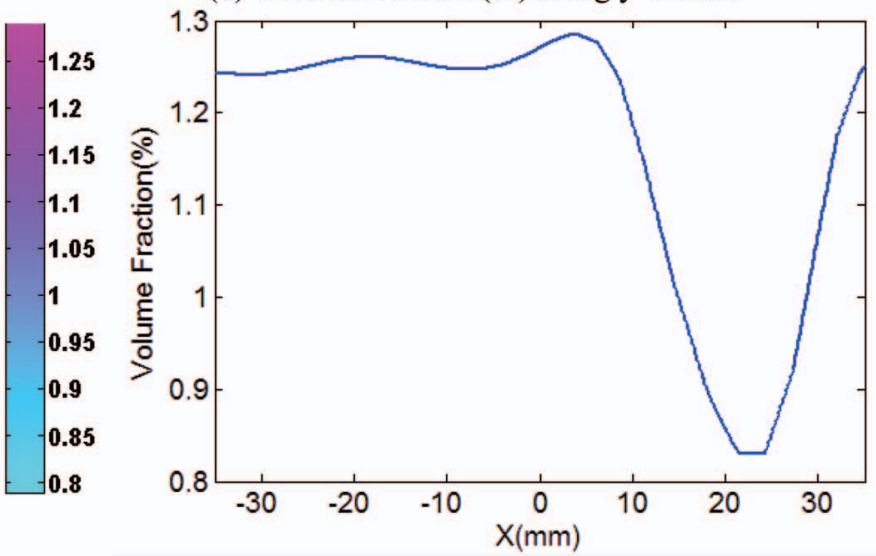

(f) Mean aspect ratio along $\mathrm{y}=0$ line.
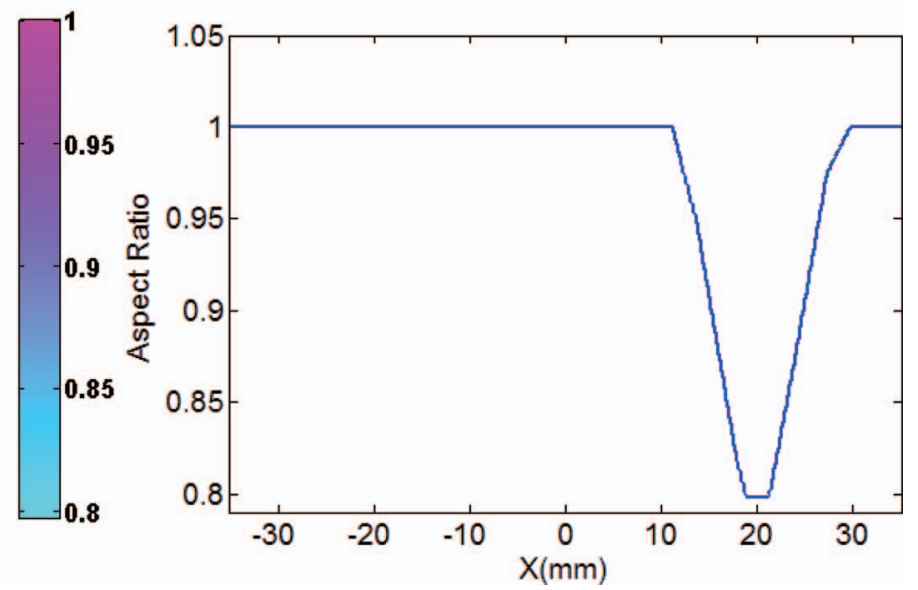

Fig. 5 (a) and (b) Reconstructed particle size, (c) and (d) concentration, and (e) and (f) aspect ratio of polystyrene prolate particles.

polymer particles (PMMA and polystyrene) are assumed to be known a priori. However, the extension of the algorithm for retrieving the refractive index is possible and is currently under development.

In Table 1, the average values of measured reduced scattering coefficient, from the MSDOT system, in the target area and the background phantom are presented. In Table 2, the measured values of particle size and aspect ratio from several microscopic images of PMMA particles are presented.

\section{Characterization of Polymer Particles}

We first tested our inverse algorithm for retrieving statistical parameters of the prolate PMMA particles. The aspect ratio was estimated to be $\sim 0.85$ for these particles from the microscopy. The equal-surface-area-sphere diameter of a prolate particle was calculated using $D_{m}=\sqrt{2 a^{2}+\frac{2 a b \xi}{\sin (\xi)}}$, where $a$ and $b$ are the equatorial and polar radius, and $\xi=\operatorname{arcos}(a / b) .{ }^{24} \mathrm{An}$ equivalent particle size of $\sim 9.1 \mu \mathrm{m}$ was estimated. Figure 3 
Table 1 Averaged reduced scattering coefficient at six wavelengths of interest $\left(\mathrm{mm}^{-1}\right)$.

\begin{tabular}{lccc}
\hline & $\begin{array}{c}\text { Example 1 } \\
\text { (Fig. 3) }\end{array}$ & $\begin{array}{c}\text { Example 2 } \\
\text { (Fig. 4) }\end{array}$ & $\begin{array}{c}\text { Example 3 } \\
\text { (Fig. 5) }\end{array}$ \\
\hline $\begin{array}{l}\text { Average } \mu_{s}^{\prime} \text { in the } \\
\text { target/inclusion }\end{array}$ & 0.122 & 0.405 & 0.315 \\
$\begin{array}{l}\text { Average } \mu_{s}^{\prime} \text { in the } \\
\text { background }\end{array}$ & 0.237 & 0.535 & 0.661 \\
\hline
\end{tabular}

presents the recovered morphologic images using our inverse algorithm where the quantitative plots along $y=0$ line (which passes through the target center) are also shown. We found that the mean particle size and aspect ratio are $8.5 \mu \mathrm{m}$ and 0.84 , respectively, in good agreement with the expected values. The concentration images [Figs. 3(c) and 3(d)] demonstrate almost two times contrast in volume fraction between the target area and the background region. The actual concentration of PMMA particles in the target area is hard to accurately estimate since the particles are recovered after chemical degradation of PDMS strips. However, the reconstructed value for the volume fraction $(\sim 1 \%)$ is close to the original concentration of the purchased particles with similar scattering spectra. In the background region, the retrieved mean particle size, volume fraction, and aspect ratio are $160 \mathrm{~nm}, 0.7 \%$, and $\sim 1$, respectively, which are in good agreement with the actual parameters of the Intralipid particles. ${ }^{6,23}$

We also conducted an experiment using the original spherical particles to demonstrate the sensitivity of the algorithm for differentiating between spherical and spheroidal particles. The images of particle size, concentration, and aspect ratio are shown in Fig. 4. We see that an aspect ratio of $\sim 1.002$ is obtained [Fig. 4(e)], which is very close to that for spherical particles. The recovered size image demonstrates a diameter close to the nominal diameter of purchased microspheres $(10 \mu \mathrm{m})$. In this experiment, since the original PMMA particles are used, we could obtain a higher concentration of particles $(\sim 2 \%$ volume fraction) as shown in Figs. 4(c) and 4(d).

As a final example, polystyrene particles having larger elongation or smaller aspect ratio compared with PMMA particles were used as the target. The reconstructed images and quantitative plots along $y=0$ line are shown in Fig. 5. Again, while the images of all three parameters are well reconstructed for this case, the recovered aspect ratio was found to be $\sim 0.79$, in good agreement with the actual value of 0.7 .

In the utilized model, the calculations are based on independently scattering particles; hence for densely packed system of

Table 2 Measured particle size and aspect ratio from five microscopic images of elongated PMMA particles.

Mean Image 1 Image 2 Image 3 Image 4 Image 5

\begin{tabular}{|c|c|c|c|c|c|c|}
\hline $\begin{array}{l}\text { Equivalent } \\
\text { diameter }(\mu \mathrm{m})\end{array}$ & 9.1 & 8.48 & 9.6 & 9.38 & 9.3 & 8.7 \\
\hline Aspect ratio & 0.85 & 0.9 & 0.83 & 0.79 & 0.91 & 0.81 \\
\hline
\end{tabular}

particles, modifications to the current model are required. The other problem is that in some cases, the Gaussian distribution model cannot accurately model the variations of the parameters; thus the employed statistical model should be replaced with other appropriate models. Furthermore, the scattering particles are assumed to be homogeneous in both target and background regions. As a result, the optical particle characterization in highly heterogeneous tissues needs some changes to the current model. Finally, when the domain under consideration is nonscattering or low scattering, the application of diffusion-equation-based diffuse optical tomography for extracting morphological parameters of particles becomes challenging and a higher-order diffusion model or radiative transfer equation based method may be needed.

We have tested the current algorithm for characterization of red blood cells which have relatively higher absorption coefficient $\left(\sim 0.1 \mathrm{~mm}^{-1}\right)$. Since this method relies on the scattering spectra, it can be employed provided that there is enough scattering contrast between the target and the background region. For highly absorbing particles, some modifications to Mishchenko's T-matrix code were suggested. ${ }^{25}$ Also, we expect that the current algorithm produces reasonable results with other tube diameters and its locations within the phantom background.

\section{Conclusions}

In conclusion, an inverse algorithm for simultaneous tomographic recovery of the size, concentration, and aspect ratio of spheroidal particles is validated using phantom experimental data. The results obtained are consistent with that measured by optical microscopy. In this work, the particle-particle interactions are not considered. Therefore, for highly dense ensembles of nonspherical particles, some modifications need to be considered in our theoretical model. We plan to report such studies in the near future.

\section{Acknowledgments}

We would like to thank Professor Mishchenko for providing the FORTRAN code used for preparing the T-matrix database. We are also grateful for Professor T. Brennan and the Particle Engineering Research Center at University of Florida for their assistance in making the nonspherical particles. This research was supported in part by NIH Grant No. R01 NS069848.

\section{References}

1. C. Li, S. R. Grobmyer, N. Massol, X. Liang, Q. Zhang, L. Chen, L. L. Fajardo, and H. Jiang, "Noninvasive in vivo tomographic optical imaging of cellular morphology in the breast: possible convergence of microscopic pathology and macroscopic radiology," Med. Phys. 35, 2493-2501 (2008).

2. A. Hsiao, M. Hunter, C. Greiner, S. Gupta, and I. Georgakoudi, "Noninvasive identification of subcellular organization and nuclear morphology features associated with leukemic cells using light-scattering spectroscopy," J. Biomed. Opt. 16, 037007 (2011).

3. G. I. Ruban, V. A. Loiko, S. M. Kosmacheva, and N. V. Goncharova, "Investigation of morphometric parameters for granulocytes and lymphocytes as applied to a solution of direct and inverse light-scattering problems," J. Biomed. Opt. 12, 044017 (2007).

4. M. Xu, T. T. Wu, and J. Y. Qu, "Unified Mie and fractal scattering by cells and experimental study on application in optical characterization of cellular and subcellular structures," J. Biomed. Opt. 13, 024015 (2008). 
5. M. G. Giacomelli, K. J. Chalut, J. H. Ostrander, and A. H. Wax, "Review of the application of T-Matrix calculations for determining the structure of cell nuclei with angle-resolved light scattering measurements," IEEE J. Sel. Top. Quantum Electron. 16(4), 900-908 (2010).

6. C. Li and H. Jiang, "Imaging of particle size and concentration in heterogeneous turbid media with multispectral diffuse optical tomography," Opt. Express 12(25), 6313-6318 (2004).

7. M. Giacomelli, Y. Zhu, J. Lee, and A. Wax, "Size and shape determination of spheroidal scatterers using two-dimensional angle resolved scattering," Opt. Express 18(14), 14616-14626 (2010).

8. X. Li, Z. Chen, A. Taflove, and V. Backman, "Equiphase-sphere approximation for analysis of light scattering by arbitrarily-shaped nonspherical particles," Appl. Opt. 43, 4497-4505 (2004).

9. V. Backman, R. Gurjar, K. Badizadegan, I. Itzkan, R. R Dasari, L. T Perelman, and M. S. Feld, "Polarized light scattering spectroscopy for quantitative measurement of epithelial cellular structures in situ," IEEE J. Sel. Top. Quantum Electron. 5(4), 1019-1026 (1999).

10. M. Kocifaj, H. Horvath, and M. Gangl, "Retrieval of aerosol aspect ratio from optical measurements in Vienna," Atmospheric Environment 42(11), 2582-2592 (2008).

11. D. D. Duncan and M. E. Thomas, "Particle shape as revealed by spectral depolarization," Appl. Opt. 46, 6185-6191 (2007).

12. C. Amoozegar, M. G. Giacomelli, J. D. Keener, K. J. Chalut, and A. Wax, "Experimental verification of T-matrix-based inverse light scattering analysis for assessing structure of spheroids as models of cell nuclei," Appl. Opt. 48, D20-D25 (2009).

13. H. Jiang, Diffuse Optical Tomography: Principles and Applications, CRC Press, Boca Raton, FL (2010).

14. C. Li, H. Zhao, B. Anderson, and H. Jiang, "Multispectral breast imaging using a ten-wavelength, $64 \times 64$ source/detector channels silicon photodiode-based diffuse optical tomography system," Med. Phys. 33, 627-636 (2006).
15. S. Jiang, B. W. Pogue, S. Davis, and K. D. Paulsen, "Multispectral NIR diffuse optical tomography system development," Biomedical Optics, Technical Digest (CD), Optical Society of America, Paper SH35 (2006).

16. Corlu, "Multi-spectral and fluorescence diffuse optical tomography of breast cancer," Ph.D. dissertation, University of Pennsylvania, Philadelphia (2007).

17. J. Tromberg, B. W. Pogue, K. D. Paulsen, A. G. Yodh, D. A. Boas, and A. E. Cerussi, "Assessing the future of diffuse optical imaging technologies for breast cancer management," Med. Phys. 35, 2443-2451 (2008).

18. H. Dehghani, B. W. Pogue, S. P. Poplack, and K. D. Paulsen, "Multiwavelength three-dimensional near-infrared tomography of the breast: initial simulation, phantom, and clinical results," Appl. Opt. 42, 135145 (2003).

19. M. I. Mishchenko, L. D. Travis, and A. A. Lacis, Scattering, Absorption, and Emission of Light by Small Particles, Cambridge University Press, Cambridge, UK (2002).

20. M. R. Hajihashemi and H. Jiang, "T-matrix-based inverse algorithm for morphologic characterization of nonspherical particles using multispectral diffuse optical tomography," Appl. Opt. 50, 3896-3906 (2011).

21. Hoekstra, V. Maltsev, and G. Videen, Optics of Biological Particles, NATO Science Series II: Mathematics, Physics and Chemistry, Springer, New York (2007)

22. K. M. Keville, E. I. Franses, and J. M. Caruthers, "Preparation and characterization of monodisperse polymer microspheroids," J. Colloid Interface Sci. 144(1), 103-126 (1991).

23. R. Michels, F. Foschum, and A. Kienle, "Optical properties of fat emulsions," Opt. Express 16, 5907-5925 (2008).

24. W. H. Beyer, CRC Standard Mathematical Tables, 28th Ed., CRC Press, Florida (1987).

25. A. Moroz, "Improvement of Mishchenko's T-matrix code for absorbing particles," Appl. Opt. 44, 3604-3609 (2005). 